\title{
Can repetitive transcranial magnetic stimulation of the visual cortex ameliorate the state of patients with visual snow?
}

\author{
Grey $\mathrm{V}^{1}$, Klobusiakova $\mathrm{P}^{1,4}$, Minks $\mathrm{E}^{1,2,3}$ \\ First Department of Neurology, Faculty of Medicine, Masaryk University and St. Anne's Hospital, \\ Brno, Czech Republic. viktoria.grey13@gmail.com
}

\begin{abstract}
AIMS: Visual snow is a neurological condition, for which an effective treatment has not been established. The aim of this study was to find whether Repetitive Transcranial Magnetic Stimulation (rTMS) can improve the state of patients suffering from visual snow. To our knowledge, no other group has tested this method in the treatment of visual snow.

METHODS: We applied rTMS of 10 and $10+1 \mathrm{~Hz}$ on the visual cortices of 9 patients with visual snow. Sham stimulation with the vertex as the target site was also tested. As a method of assessment, we used visual evoked potentials, questionnaires and visual snow diaries. For data evaluation, we used the Paired Sample T-test separately for each stimulation type.

RESULTS: The Paired Sample T-test revealed a decreased sum of visual snow intensities extracted from visual snow diaries in the week after $10+1 \mathrm{~Hz}$ stimulation as compared to the figure in the week before $(p=0.02)$.

CONCLUSION: We detected a trend indicating an improvement of patients' condition based on the data from visual snow diaries. Research on a larger group of patients is required to confirm these findings; however, our study provides a framework to build upon (Tab. 4, Fig. 1, Ref. 22). Text in PDF www.elis.sk.

KEY WORDS: visual snow, persistent aura without infarction, rTMS, occipital cortex.
\end{abstract}

Abbreviations: PAWI - persistent aura without infarction, rTMS repetitive transcranial magnetic stimulation, $\mathrm{HF}$ - high-frequency, LF - low-frequency, VEP - visual evoked potentials, MEP - motor evoked potentials.

\section{Introduction}

Persistent aura without infarction (PAWI, also known as Persistent Migraine Aura - PMA) is a rare neurological condition described in the International Classification of Headache Disorders ICHD-3 as a complication of migraine (number 1.4.2). PAWI is defined by the presence of aura symptoms persisting for one week or more without any signs of brain infarction on neuroimaging (1). These so-called aura symptoms are persistent visual disturbances, often called visual snow. They are described by patients as "speckling, combined with flashing or colour effects", "seeing the world as though from behind a veil" or "television static" (2). Patients might also experience other symptoms such as: palinopsia (persistent recurrence of a visual image, after the stimulus has been removed), photophobia and nyctalopia (impaired night vision) (3).

${ }^{1}$ First Department of Neurology, Faculty of Medicine, Masaryk University and St. Anne's Hospital, Brno, Czech Republic, ${ }^{2}$ Neurology and Physiotherapy Outpatient Clinic Skopalíkova, Brno, Czech Republic, ${ }^{3}$ Neurology, TERAneuro, s.r.o., Židlochovice, Czech Republic, and ${ }^{4}$ CEITEC, Masaryk University, Kamenice, Brno, Czech Republic

Address for correspondence: V. Grey, First Department of Neurology, St. Anne's Hospital, Brno, Pekarska 53, CZ-656 91 Brno, Czech Republic.
A specific classification of PAWI has been proposed, dividing PAWI patients into two different sub-groups - PAWI with typical aura and PAWI with persistent visual disturbance. The first group of patients experiences their aura as being very similar to the classic migraine aura, only lasting much longer. The second group of patients describes the visual snow as mentioned above $(4,5)$. In our study, we dealt with PAWI patients describing their pathological symptoms in accordance with visual snow symptoms. Lately, there have also been attempts by several authors to completely separate so-called "Visual Snow Syndrome" from Persistent Aura Without Infarction (6).

It is currently not known how many people suffer from visual snow worldwide, but from available data it is possible to say that on average the population diagnosed with this condition is male and relatively young (3). The condition that is strongly associated with visual snow is tinnitus - several studies indicate a shared pathology $(7,8)$.

Migraine attacks are less frequent in these patients and do not usually cause any trouble (2). Visual snow has a negative impact on the physical and mental well-being of the patients (9). In PAWI diagnostics it is necessary to exclude other pathological conditions, which could have similar clinical manifestations - specifically migraine infarction and vitreous opacities, visual disturbances induced by hallucinogens, disturbances accompanying mental disorders, neurological infections, focal organic lesions in the brain, sclerosis multiplex, brain tumours and others. The exclusion of these conditions is very important, because 
there have been cases, where visual snow syndrome did have an organic background (10).

An effective treatment for PAWI remains to be found. All available data on the treatment of visual snow comes from single case reports (3). Several pharmacological possibilities routinely used for migraine treatment could have a beneficial effect. However, among our patients, Valproate, Topiramate and Lamotrigine have only had a positive influence on migraine attacks and not on visual snow.

Transcranial Magnetic Stimulation (TMS) is a non-invasive diagnostic and therapeutic method based on the use of a magnetic field of the order of several Teslas. The magnetic field is transmitted through a special coil enclosing the patient's head and activates different neural pathways through the depolarisation of superficial axons (11). While Paired-pulse TMS is used for measuring cortex excitability, Repetitive Transcranial Magnetic Stimulation is strictly a therapeutic method. In rTMS, a large number of magnetic pulses are applied to the patient's brain cortex. We know two basic types of rTMS, based on the frequency of pulses - low-frequency rTMS (LF rTMS) and high-frequency rTMS (HF rTMS). rTMS is well studied as method, which is used to influence cortex excita bility (12). rTMS is currently being studied in the treatment of a wide group of pathologies and is not limited to the stimulation of the neocortex, but can also be used for stimulation of cerebellum (13).

So far, there have not been any studies dealing with PAWI and rTMS. However, as we have already mentioned in our review article (14), PAWI and classic migraine aura have some similarities $(15,16)$. Due to this fact, we have decided to go through literature concerning migraine aura and its treatment with rTMS. It has been proposed by Mullener et al 2001 that the occipital cortices of patients suffering from migraine aura were hyperexcitable (17). It has also been shown that LF rTMS played an important role in reducing visual cortex excitability in healthy patients (18). However, LF rTMS has been shown paradoxically to increase excitability in migraine patients, probably due to a dysfunction in the cortical GABA-mediated circuits $(19,20)$. In the later study, the same author decided to try HF rTMS as another possibility that could improve the state of migraine aura patients. The combination of HF rTMS and subsequent LF rTMS was effective in reducing visual cortex excitability (21). Therefore, we tried to use a similar protocol in the treatment of our visual snow patients in order to see if we could improve their state.

\section{Subjects and methods}

The study protocol was approved by the Ethics Committee of St. Anne's Hospital in Brno and conformed to the Declaration of Helsinki. All participants gave their written informed consent and there were no adverse events during our study.

\section{Subjects}

Nine patients participated in our study, three women and six men (age $32.6 \pm 9.1$ ), altogether in 20 rTMS sessions. All participants had a positive visual snow history and underwent MRI scans of their brains, which showed no brain pathology. Also,
Tab. 1. Description of the group of subjects.

\begin{tabular}{cccc}
\hline Patients & Sex & Age (years) & Visual snow duration \\
\hline 1 & M & 50 & 6 years \\
2 & F & 35 & 4 years \\
3 & F & 35 & 2 years \\
4 & F & 41 & 2 years \\
5 & M & 19 & from childhood \\
6 & M & 26 & 1 year \\
7 & M & 30 & 15 years \\
8 & M & 29 & from childhood \\
9 & M & 28 & 1 year \\
\hline
\end{tabular}

other methods of examination, such as: ophthalmological examination, electroencephalography, lumbar puncture, psychological and psychiatric examination did not clarify the cause of patients' problems. Only two of our participants referred to a history of migraine attacks with aura. These attacks were rare (one in several months), but of a strong intensity. Visual snow was described by the patients as television static, flashing, sometimes speckling combined with different colour effects. Two of the patients also experienced palinopsia, the blue-field phenomenon or the vision of halos around objects. Two participants also referred to the presence of tinnitus (Tab. 1).

\section{Protocol}

rTMS was applied to the primary visual cortex of our patients. One session of rTMS consisted of two separate applications - on the first day to the left visual cortex and on the second to the right visual cortex. We used two different protocols in our study. In the first protocol, rTMS of $10 \mathrm{~Hz}$ was used. The length of each train was 3 seconds, while the interstimulus interval was 10 seconds. The overall time of rTMS stimulation was 13 minutes and it consisted of 1800 pulses. The second protocol consisted both of $10 \mathrm{~Hz}$ and $1 \mathrm{~Hz}$ rTMS. We decided to add the low-frequency stimulation to the second protocol in order to inhibit the hyperexcitable visual cortices of the patients. As for the first $10 \mathrm{~Hz}$ sequence, the protocol remained the same (1800 pulses in 13 minutes). The $1 \mathrm{~Hz}$ part consisted of 900 pulses applied in 15 minutes. The overall length of the new protocol was then 28 minutes. In order to objectify our results, we decided to use also sham (placebo) stimulation. In the sham stimulation, the rTMS parameters remained the same, but

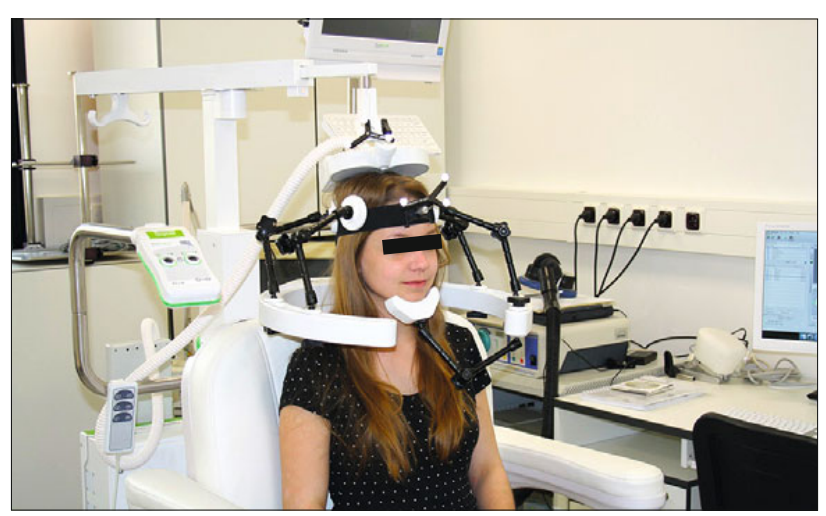

Fig. 1. rTMS device. 
we changed the stimulation site. For an eligible sham stimulation site, we chose the vertex (22). The first protocol consisting simply of $10 \mathrm{~Hz}$ rTMS was used in 6 sessions, the $10+1 \mathrm{~Hz}$ one was used in 9 sessions, and sham stimulation was performed in 5 remaining sessions out of 20 .

\section{Stimulation site}

The exact spots for rTMS application were defined on the MRI scans of the patient's brain. We orientated according to the sulcus calcarinus and determined the visual cortex for each hemisphere before stimulation. For the stimulation, we used a DuoMAG TM XT-100 Magnetic Stimulator (stimulation software Brain Sight). With the help of the navigation system, the position of the patients' head was recorded by the camera in real time and integrated with the 3-D model of the patient's brain. The stimulation coil was then navigated to the exact stimulation target and the protocol was triggered. Before each session, the motor threshold of the patient was defined using motor evoked potentials (MEP) stimulating the motor cortex for the first dorsal interosseous muscle. Afterwards, we stimulated using $90 \%$ of the motor threshold intensity.

\section{Evaluation}

We used three different methods for the evaluation - visual snow diaries, questionnaires and finally visual evoked potentials (VEP).

\section{Visual snow diaries}

Diaries were the optimal method of evaluating patients' subjective state. Each day the patients scored the intensity of their visual snow with a number from 1 to 5 ; 1 being the lowest and 5 the highest intensity. They also recorded their headache and migraine attacks and the presence or absence of aura before the migraine attack. We managed to collect data from eighty-five per cent of visual snow diaries. Visual snow intensities were extracted from visual snow diaries. In order to compare the overall intensities during the week before rTMS sessions to the intensities during the week immediately after rTMS sessions, intensities within each week were summed and compared using the Paired Sample T-test separately for each stimulation type. With respect to specific intensities distribution before and after the stimulation, these were divided into the two categories - low (1-3) and high intensities (4-5). For each stimulation type, a contingency table (before/after and low intensities/high intensities) was created and Pearson's Chi-squared test was applied. The significance level was set to 0.05 . In order to compare active stimulation to sham in terms of improving visual snow diaries scores, we used repeated ANOVA measures with time (before and after stimulation) as a within subject factor, and stimulation type (sham, $10 \mathrm{~Hz}$ or $10+1 \mathrm{~Hz}$ ) as a between subject factor.

\section{Questionnaires}

The second method of evaluation was questionnaires, which recorded the influence of visual snow and headache on patients' common daily activities such as: working, studying, household chores and participating in family and social events. The questions were posed in order to find out how many days during the
Tab. 2. Extracted visual snow intensities from the diaries - week before the stimulation.

\begin{tabular}{lllllllll}
\hline $\begin{array}{l}\text { Session } \\
\text { number }\end{array}$ & Protocol & \multicolumn{7}{c}{ Visual snow intensity $(1-5)-$ before the stimulation } \\
\hline 1 & $10 \mathrm{~Hz}$ & 3 & 2 & 2 & 2 & 3 & 2 & 3 \\
2 & $10 \mathrm{~Hz}$ & 3 & 3 & 3 & 3 & 3 & 3 & 3 \\
3 & $10 \mathrm{~Hz}$ & 2 & 1 & 1 & 2 & 1 & 2 & 1 \\
4 & $10 \mathrm{~Hz}$ & 3.5 & 3.5 & 3.5 & 4 & 3 & 4 & 3 \\
5 & sham & 1 & 2 & 1 & 2 & 2 & 1 & 1 \\
6 & sham & 4 & 3 & 3 & 3 & 4 & 3.5 & 4 \\
7 & $10 \mathrm{~Hz}$ & 5 & 5 & 5 & 5 & 5 & 5 & 5 \\
8 & $10+1 \mathrm{~Hz}$ & 4 & 3 & 4 & 4 & 4 & 4 & 4 \\
9 & $10+1 \mathrm{~Hz}$ & 5 & 5 & 5 & 1 & 1 & 1 & 1 \\
10 & $10+1 \mathrm{~Hz}$ & 3 & 3 & 3 & 3 & 3 & 3 & 3 \\
11 & $10+1 \mathrm{~Hz}$ & 4 & 4 & 4 & 4 & 4 & 4 & 4 \\
12 & $10+1 \mathrm{~Hz}$ & 5 & 5 & 5 & 5 & 5 & 5 & 5 \\
13 & sham & 1 & 3 & 1 & 3 & 3 & 3 & 3 \\
14 & sham & 3 & 4 & 4.5 & 4 & 4 & 3 & 3 \\
15 & sham & 3 & 3 & 3 & 3 & 3 & 3 & 3 \\
16 & $10+1 \mathrm{~Hz}$ & 3 & 3 & 3 & 3 & 3 & 3 & 3 \\
17 & $10+1 \mathrm{~Hz}$ & 5 & 5 & 5 & 5 & 5 & 5 & 5 \\
\hline
\end{tabular}

Tab. 3. Extracted visual snow intensities from the diaries - week after the stimulation.

\begin{tabular}{cllllllll}
\hline $\begin{array}{c}\text { Session } \\
\text { number }\end{array}$ & Protocol & \multicolumn{7}{c}{ Visual snow intensity $(1-5)-$ after the stimulation } \\
\hline 1 & $10 \mathrm{~Hz}$ & 2 & 2 & 2 & 2 & 2 & 3 & 3 \\
2 & $10 \mathrm{~Hz}$ & 3 & 3 & 3 & 3 & 3 & 3 & 3 \\
3 & $10 \mathrm{~Hz}$ & 1 & 2 & 1 & 2 & 2 & 1 & 1 \\
4 & $10 \mathrm{~Hz}$ & 3 & 3 & 3.5 & 4 & 4 & 4 & 4 \\
5 & sham & 2 & 2 & 1 & 1 & 1 & 2 & 2 \\
6 & sham & 3 & 3 & 3 & 3 & 3 & 3 & 3 \\
7 & $10 \mathrm{~Hz}$ & 5 & 5 & 5 & 5 & 5 & 5 & 5 \\
8 & $10+1 \mathrm{~Hz}$ & 4 & 3 & 3 & 3 & 3 & 3 & 3 \\
9 & $10+1 \mathrm{~Hz}$ & 1 & 3 & 1 & 1 & 1 & 1 & 3 \\
10 & $10+1 \mathrm{~Hz}$ & 3 & 3 & 3 & 3 & 3 & 3 & 3 \\
11 & $10+1 \mathrm{~Hz}$ & 3 & 3 & 3 & 3 & 3 & 3 & 4 \\
12 & $10+1 \mathrm{~Hz}$ & 5 & 5 & 5 & 5 & 5 & 5 & 5 \\
13 & sham & 1 & 1 & 1 & 3 & 1 & 3 & 1 \\
14 & sham & 3 & 4 & 3 & 4 & 3 & 3 & 4 \\
15 & sham & 3 & 3 & 3 & 3 & 3 & 3 & 3 \\
16 & $10+1 \mathrm{~Hz}$ & 3 & 3 & 3 & 3 & 3 & 3 & 3 \\
17 & $10+1 \mathrm{~Hz}$ & 5 & 4 & 5 & 5 & 5 & 5 & 4 \\
\hline
\end{tabular}

last three months the patient's productivity at work, school or in the household was decreased because of visual snow (questionnaire 1) and headache (questionnaire 2). We managed to collect questionnaires from seventy per cent of all sessions. The numbers of the days three months before and three months after the rTMS session was compared. The scores from questionnaires - the number of days the patient was influenced by visual snow or headache - were first compared day-to-day and then also the sums of individual days' scores before and after stimulation using the Paired Sample T-test, separately for each stimulation type. FDR correction with a critical expected False Discovery Rate set to 0.05 was applied.

\section{Visual evoked potentials}

The last method of evaluation was Visual Evoked Potentials (VEP). VEP is the measurement of the electrical activity of the visual pathway recorded by electrodes connected to the scalp. VEP in our study was recorded immediately before the first rTMS ap- 
Tab. 4. Results of visual snow diaries analysis.

\begin{tabular}{lr}
\hline Stimulation type & $\mathrm{p}$ \\
\hline sham & 0.19 \\
$10 \mathrm{~Hz}$ & 1 \\
$10+1 \mathrm{~Hz}$ & 0.05 \\
\hline
\end{tabular}

plication and immediately after the second rTMS application the next day. This means that there were two VEP measurements in one rTMS session. The VEP values before and after rTMS were compared using the Paired Sample T-test, separately for each stimulation type. We looked for the changes in the visual pathway and visual cortex functioning that could be caused by the impact of rTMS on the visual cortex. We compared altogether 6 parameters from the main lead both for the left and right side VEP. These parameters included Latency of the N75 wave, P100 wave and N145 wave (ms) and Voltage of the P100 wave $(\mu \mathrm{V})$, Duration of the P100 wave (ms) and Area over the curve ( $\mu \mathrm{Vms})$. To correct for multiple comparisons, we used False Discovery Rate (FDR) correction with the critical expected FDR set to 0.05 .

All statistical tests were calculated using Matlab 2014b (Tabs $2,3)$.

\section{Results}

\section{Visual snow diaries}

The Paired Sample T-test revealed a trend for a reduced sum of visual snow intensities in the week after $10+1 \mathrm{~Hz}$ stimulation as compared to the sum of visual snow intensities in the week before $(\mathrm{p}=0.05)$. When testing sham and $10 \mathrm{~Hz}$ stimulation, no significant effect was observed ( $\mathrm{p}=0.19, \mathrm{p}=1$; respectively). Pearson's Chi-squared test comparing low and high intensities distribution before and after stimulation showed a higher number of low intensities and a lower number of high intensities after $10+1 \mathrm{~Hz}$ stimulation $\left(\mathrm{x}^{2}=8.03, \mathrm{p}=0.05\right)$, whereas this shift was not significant after sham $\left(x^{2}=2.7, p=0.1\right)$ or $10 \mathrm{~Hz}$ stimulation $\left(x^{2}=0, p=1\right)$. However, the interaction between time and stimulation type was not significant $(\mathrm{p}=0.19)$.

\section{Questionnaires}

No significant changes in the questionnaire scores after stimulation were observed in comparison to the scores before rTMS stimulation.

\section{Visual evoked potentials}

No significant changes in VEP parameters were observed (Tab. 4).

\section{Discussion}

This study was designed to find a new therapy for patients suffering from visual snow, because effective therapies are very limited. The main problem we had to face, when designing the stimulation protocol, was a lack of adequate data. There have not been any studies dealing with rTMS in visual snow patients to date. As we have already mentioned in the introduction, we relied on the studies that tried rTMS in patients with migraine aura, when forming the stimulation protocol $(18,21)$, because PAWI and classic migraine aura share some similarities in pathology $(15,16)$.

Our study did not avoid certain limitations. The main limitation was the low number of the subjects altogether and also in the specific types of rTMS stimulation. The next limitation was the fact that we stimulated nine subjects in 20 sessions, which means that some of the subjects had more than one session and the number of sessions for each subject differed. The other undeniable shortcoming of our study was that we did not manage to collect data from diaries and questionnaires for all of the sessions. This means that there were three sessions for which visual snow diaries were not documented (Table 2 - only 17 sessions) and six sessions for which questionnaires were not well documented. This was caused by patients' non-compliance. Whether this non-compliance can be partly attributed to illness is difficult to say. Patients were fully informed of the importance of their cooperation in the study. Despite the fact that we originally wanted to expand the group of patients, the negative preliminary results altogether with our limited capacity resulted in the study termination.

In our study results, we recorded a certain trend in decreasing sum of visual snow intensities after $10+1 \mathrm{~Hz}$ rTMS stimulation $(p=0.05)$. This might indicate that the stimulation of $10+1 \mathrm{~Hz}$ had a positive effect on patients' visual cortices and ameliorated their state. Patients' subjective scoring of visual snow during the week after $10+1 \mathrm{~Hz}$ rTMS stimulation was lower than during the week preceding this stimulation. However, due to the earlier mentioned limitations, mainly the low number of subjects included in $10+1 \mathrm{~Hz}$ stimulation, it is difficult to say whether this result is reliable. Still, this positive trend shows that there might be a certain hope in the future treatment of patients with visual snow. The application of rTMS for more than two days on a larger sample of patients would be needed to verify this statement. Because we have been the first to apply the rTMS protocol in visual snow patients, we went for a rather cautious method of stimulation - only two successive days per session and a three-month pause between individual sessions. It cannot be excluded that with a longer lasting rTMS protocol, results would show a more significant trend. In our study, we focused on the precision of rTMS application. With precise rTMS navigation together with manual and double-checked specification of the stimulation point - the visual cortex - we have been able to apply rTMS reliably and safely each time. The change in stimulation protocol from $10 \mathrm{~Hz}$ to $10+1 \mathrm{~Hz}$ was done to more closely resemble the 2009 study by Brighina et al, where the same combination of a $10 \mathrm{~Hz}$ and subsequent $1 \mathrm{~Hz}$ protocol was used (21).

Although our study was limited, we wish to point out the complexity of the study design and several mistakes that have been made, so that authors in future can improve the design and avoid the same shortcomings. Further research is needed in this area with a wider sample of subjects.

\section{Conclusion}

A two-day session of rTMS of right and left occipital cortex with a frequency of $10+1 \mathrm{~Hz}$ lowered the sum of visual snow in- 
tensities during the week after the stimulation, as proved by the trend in Paired Sample T-test. The results in other methods of assessment (VEP and questionnaires) did not show any significant trend. We presume that rTMS could present a potential method of treatment for patients suffering from visual snow in future, after more research is completed and after a sufficient evidence is provided in this field. Changes in protocol in the matter of rTMS session length and size of patient group should be considered.

\section{References}

1. Headache Classification Committee of the International Headache Society (IHS) The International classification of Headache Disorders. Cephalalgia 2018; 38 (1): 1-211.

2. Mastík MudJ. Perzistující migrenózní aura bez infarktu. [cited 2016 Jul 17]; Available from: http: //www.neurologiepropraxi.cz/savepdfs/ neu/2012/01/04.pdf.

3. Puledda F, Schankin C, Digre K, Goadsby PJ. Visual snow syndrome: what we know so far. Curr Opin Neurol 2018; 31 (1): 52-58.

4. Mechanism and Management of Headache - 7th Edition [Internet]. [cited 2019 Jan 21]. Available from: https: //www.elsevier.com/books/ mechanism-and-management-of-headache/lance/978-0-7506-7530-7.

5. Plato BM. Rare Complications of Migraine With Aura. Headache J Head Face Pain 2016; 56 (8): 1373-1379.

6. Metzler AI, Robertson CE. Visual Snow Syndrome: Proposed Criteria, Clinical Implications, and Pathophysiology. Curr Neurol Neurosci Rep 2018; 18 (8): 52.

7. Renze M. Visual Snow Syndrome and Its Relationship to Tinnitus. Int Tinnitus J 2017; 21 (1): 74-75.

8. Puledda F, Tze L, Schankin C, Goadsby P. PO069 Clinical characterisation of visual snow. J Neurol Neurosurg Psychiatry 2017; 88 (Suppl 1): $\mathrm{A} 30-\mathrm{A} 30$.

9. Bou Ghannam A, Pelak VS. Visual Snow: a Potential Cortical Hyperexcitability Syndrome. Curr Treat Options Neurol 2017; 19 (3): 9.

10. Chen BS, Lance S, Lallu B, Anderson NE. Visual snow: Not so benign. J Clin Neurosci Off J Neurosurg Soc Australas 2019; 64: 37-39.

11. Minks E, Kopickova M, Marecek R, Streitova H, Bares M. Transcranial magnetic stimulation of the cerebellum. Biomed Pap Med Fac Univ Palacky Olomouc Czechoslov 2010; 154 (2): 133-139.
12. Lefaucheur J-P, André-Obadia N, Antal A, Ayache SS, Baeken C, Benninger DH, et al. Evidence-based guidelines on the therapeutic use of repetitive transcranial magnetic stimulation (rTMS). Clin Neurophysiol 2014; 125 (11): 2150-2206.

13. Minks E, Mareček R, Pavlík T, Ovesná P, Bareš M. Is the cerebellum a potential target for stimulation in Parkinson's disease? Results of 1-Hz rTMS on upper limb motor tasks. Cerebellum Lond Engl 2011; 10 (4): 804-811.

14. Lukáčová V, Mastík J, Minks E. Could Repetitive Transcranial Magnetic Stimulation (rTMS) Help Patients with Visual Snow? Act Nerv Super (Praha) 2018; 60 (1): 27-31.

15. Schankin CJ, Goadsby PJ. Visual Snow - Persistent Positive Visual Phenomenon Distinct from Migraine Aura. Curr Pain Headache Rep 2015; 19 (6): 23.

16. Chen WT, Lin YY, Fuh JL, Hämäläinen MS, Ko YC, Wang SJ. Sustained visual cortex hyperexcitability in migraine with persistent visual aura. Brain 2011; 134 (8): 2387-2395.

17. Mulleners WM, Chronicle EP, Palmer JE, Koehler PJ, Vredeveld JW. Visual Cortex Excitability in Migraine with and without Aura. Headache J Head Face Pain 2001; 41 (6): 565-572.

18. Boroojerdi B, Prager A, Muellbacher W, Cohen LG. Reduction of human visual cortex excitability using $1-\mathrm{Hz}$ transcranial magnetic stimulation. Neurology 2000; 54 (7): 1529-1531.

19. Brighina F, Piazza A, Daniele O, Fierro B. Modulation of visual cortical excitability in migraine with aura: effects of $1 \mathrm{~Hz}$ repetitive transcranial magnetic stimulation. Exp Brain Res 2002; 145 (2): 177-181.

20. Galeotti N, Ghelardini C, Zoppi M, Del Bene E, Raimondi L, Beneforti $\mathbf{E}$, et al. Hypofunctionality of Gi proteins as aetiopathogenic mechanism for migraine and cluster headache. Cephalalgia Int J Headache 2001; 21 (1): $38-45$.

21. Brighina F, Palermo A, Daniele O, Aloisio A, Fierro B. High-frequency transcranial magnetic stimulation on motor cortex of patients affected by migraine with aura: a way to restore normal cortical excitability? Cephalalgia [Internet] 2009 May [cited 2016 Jul 17]; Available from: http: //cep.sagepub.com/cgi/doi/10.1111/j.1468-2982.2009.01870.x

22. Pitcher D, Walsh V, Yovel G, Duchaine B. TMS evidence for the involvement of the right occipital face area in early face processing. Curr Biol CB 2007; 17 (18): 1568-1573.

Received December 19, 2019. Accepted February 3, 2020. 